\title{
ScientoMiner ICR - the Gephi plugin for importing scholarly citations data from Crossref services
}

\author{
Nataliia Kaliuzhna
}

Potential competing interests: The author(s) declared that no potential competing interests exist.

The proposed module ScientoMiner ICR for the Gephi platform simplifies the process of automatic export of Crossref data directly to Gephi, in particular, for the needs of bibliometric analysis. Link to the presentation: https://zenodo.org/record/3888138 